Matthes, Eva

\title{
Geisteswissenschaftliche Pädagogik und höhere Lehrerausbildung
} (1915-1960)

Casale, Rita [Hrsg.]; Windheuser, Jeannette [Hrsg.]; Ferrari, Monica [Hrsg.]; Morandi, Matteo [Hrsg.]: Kulturen der Lehrerbildung in der Sekundarstufe in Italien und Deutschland. Nationale Formate und 'cross culture'. Bad Heilbrunn : Verlag Julius Klinkhardt 2021, S. 177-190. - (Historische Bildungsforschung)

Quellenangabe/ Reference:

Matthes, Eva: Geisteswissenschaftliche Pädagogik und höhere Lehrerausbildung (1915-1960) - In: Casale, Rita [Hrsg.]; Windheuser, Jeannette [Hrsg.]; Ferrari, Monica [Hrsg.]; Morandi, Matteo [Hrsg.]: Kulturen der Lehrerbildung in der Sekundarstufe in Italien und Deutschland. Nationale Formate und 'cross culture'. Bad Heilbrunn : Verlag Julius Klinkhardt 2021, S. 177-190 - URN: urn:nbn:de:0111-pedocs-225861 - DOI: 10.25656/01:22586

https://nbn-resolving.org/urn:nbn:de:0111-pedocs-225861

https://doi.org/10.25656/01:22586

in Kooperation mit / in cooperation with:

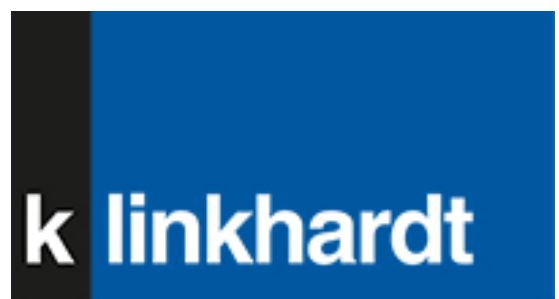

http://www.klinkhardt.de

\section{Nutzungsbedingungen}

Dieses Dokument steht unter folgender Creative Commons-Lizenz: http://creativecommons.org/licenses/by-nc-nd/4.0/deed.de - Sie dürfen das Werk bzw. den Inhalt unter folgenden Bedingungen vervielfältigen, verbreiten und öffentlich zugänglich machen: Sie müssen den Namen des Autors/Rechteinhabers in der von ihm festgelegten Weise nennen. Dieses Werk bzw. dieser Inhalt darf nicht für kommerzielle Zwecke verwendet werden und es darf nicht bearbeitet, abgewandelt oder in anderer Weise verändert werden.

Mit der Verwendung dieses Dokuments erkennen Sie die Nutzungsbedingungen an.

\section{Terms of use}

This document is published under following Creative Commons-License: http://creativecommons.org/licenses/by-nc-nd/4.0/deed.en - You may copy, distribute and transmit, adapt or exhibit the work in the public as long as you attribute the work in the manner specified by the author or licensor. You are not allowed to make commercial use of the work or its contents. You are not allowed to alter, transform, or change this work in any other way.

By using this particular document, you accept the above-stated conditions of use.

\section{Kontakt / Contact:}

\section{peDOCS}

DIPF | Leibniz-Institut für Bildungsforschung und Bildungsinformation Informationszentrum (IZ) Bildung

E-Mail: pedocs@dipf.de

Internet: www.pedocs.de

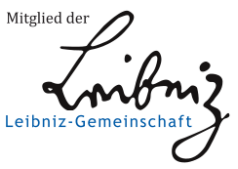




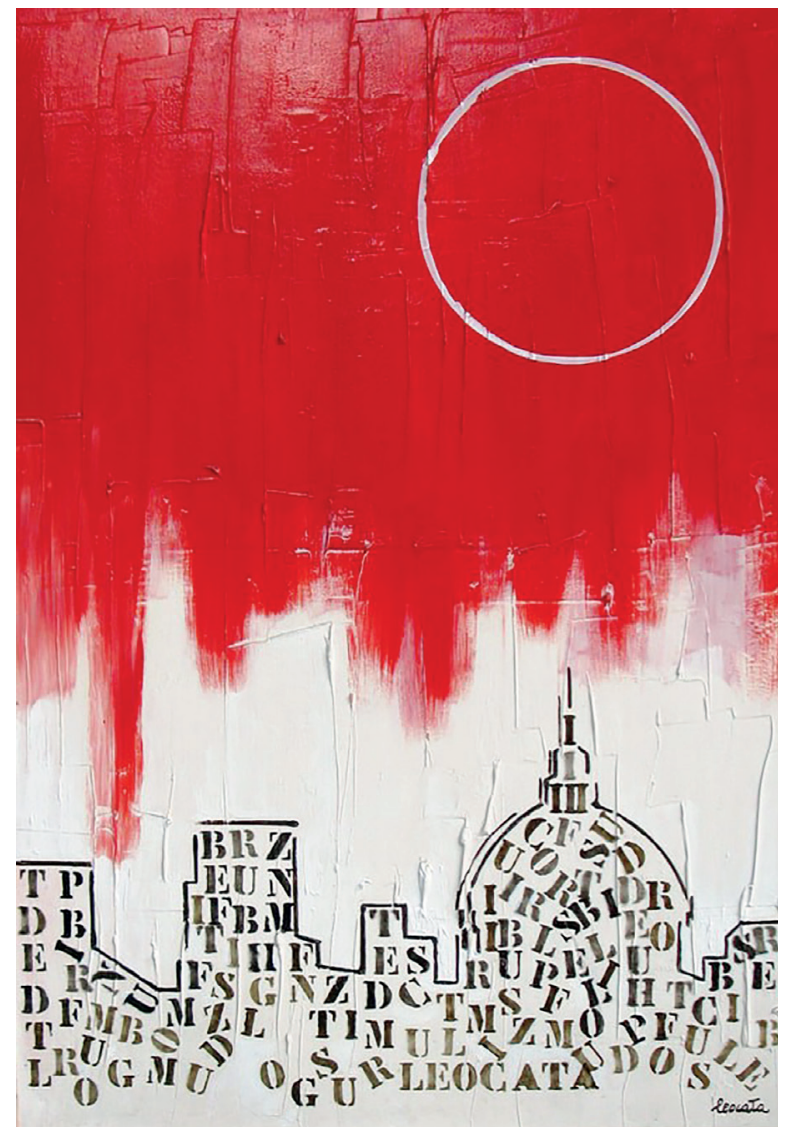

Rita Casale / Jeannette Windheuser Monica Ferrari / Matteo Morandi (Hrsg.)

\section{Kulturen der Lehrerbildung in der Sekundarstufe in Italien und Deutschland}

Nationale Formate und ,cross culture، 
Casale / Windheuser / Ferrari / Morandi

Kulturen der Lehrerbildung in der Sekundarstufe in Italien und Deutschland 


\section{Historische Bildungsforschung}

herausgegeben von

Rita Casale, Ingrid Lohmann und Eva Matthes

\section{In dieser Reihe sind erschienen}

Vogt, Michaela: Professionswissen über Unterstufenschüler in der DDR. Untersuchung der Lehrerzeitschrift „Die Unterstufe“ im Zeitraum 1954 bis 1964. Bad Heilbrunn 2015.

Heinemann, Rebecca: Das Kind als Person. William Stern als Wegbereiter der Kinder- und Jugendforschung 1900 bis 1933. Bad Heilbrunn 2016.

Conrad, Anne/Maier, Alexander (Hrsg.): Erziehung als ,Entfehlerung'. Weltanschauung, Bildung und Geschlecht in der Neuzeit. Bad Heilbrunn 2017.

Müller, Ralf: Die Ordnung der Affekte. Frömmigkeit als Erziehungsideal bei Erasmus von Rotterdam und Philipp Melanchthon. Bad Heilbrunn 2017.

Zimmer, Eva: Wandbilder für die Schulpraxis. Eine historisch-kritische Analyse der Wandbildproduktion des Verlags Schulmann 1925-1987. Bad Heilbrunn 2017.

Horn, Elija: Indien als Erzieher. Orientalismus in der deutschen Reformpädagogik und Jugendbewegung 1918-1933. Bad Heilbrunn 2018.

Haupt, Selma: Das Beharren der Rektoren auf die „Deutsche Universität“. Bad Heilbrunn 2018. Wehren, Sylvia: Erziehung - Körper - Entkörperung. Forschungen zur pädagogischen Theorieentwicklung. Bad Heilbrunn 2020. 


\section{Rita Casale Jeannette Windheuser Monica Ferrari Matteo Morandi \\ (Hrsg.)}

\section{Kulturen der Lehrerbildung in der Sekundarstufe in Italien und Deutschland}

Nationale Formate und ,cross culture 
Der vorliegende Band konnte mithilfe der finanziellen Unterstützung des Lehrstubls für Allgemeine Erziehungswissenschaft / Theorie der Bildung der Bergischen Universität Wuppertal veröffentlicht werden.

Aufgrund einer Vereinbarung zwischen den beiden Verlagen, die die gegenseitige freie Verfügbarkeit der Vertriebsrechte festlegt, erscheint dieser Band zeitgleich in Italien im Verlag FrancoAngeli, herausgegeben von M. Ferrari, M. Morandi, R. Casale und J. Windheuser, unter dem Titel La formazione degli insegnanti della secondaria in Italia e in Germania. Una questione culturale.

Die Herausgeber*innen bedanken sich bei dem Künstler Pippo Leocata für die kostenfreie Überlassung des Titelbildes.

Dieser Titel wurde in das Programm des Verlages mittels eines Peer-Review-Verfahrens aufgenommen. Für weitere Informationen siehe www.klinkhardt.de.

Bibliografische Information der Deutschen Nationalbibliothek Die Deutsche Nationalbibliothek verzeichnet diese Publikation in der Deutschen Nationalbibliografie; detaillierte bibliografische Daten sind im Internet abrufbar über http://dnb.d-nb.de.

2021.lg. ( ) by Julius Klinkhardt.

Grafik Umschlagseite 1: () by Pippo Leocata, La città delle parole [Die Stadt der Worte], Öl und Acryl auf Leinwand, 2014. (Privatsammlung)

Druck und Bindung: Bookstation GmbH, Anzing.

Printed in Germany 2021.

Gedruckt auf chlorfrei gebleichtem alterungsbeständigem Papier.

Die Publikation (mit Ausnahme aller Fotos, Grafiken und Abbildungen) ist veröffentlicht unter der Creative Commons-Lizenz: CC BY-NC-ND 4.0 International https://creativecommons.org/licenses/by-nc-nd/4.0/ 


\section{Inhaltsverzeichnis}

\section{Einführung}

Nationale Fälle und cross culture in der Lehrerbildung

Rita Casale, Monica Ferrari, Matteo Morandi und Jeannette Windheuser .7

\section{Italien}

Habsburg Legislation on the Training of Elementary and Ginnasio-Liceo (Secondary) Teachers and its Implementation in the Italian Territories across the $18^{\text {th }}$ and $19^{\text {th }}$ Centuries

Simonetta Polenghi

The Scuola Normale Superiore di Pisa: between French Model and Autonomous Choices (1810-1923)

Paola Carlucci und Mauro Moretti

Long-Term Issues in Secondary School Teacher Training in Italy

(1862-2015)

Matteo Morandi

Belgium as a Cultural Model for Building the Italian Secondary School and Training its Teachers (1860-1900)

Mara Donato di Paola

An Atypical Path of Women Teachers Training, between the $19^{\text {th }}$ and $20^{\text {th }}$ Centuries: the Istituti Superiori di Magistero femminile

(Higher Institutes of Teaching for Women)

Tiziana Pironi

The Professor of Pedagogy and Italian Textbooks between the $19^{\text {th }}$ and the $20^{\text {th }}$ Centuries

Giuseppe Zago

Pedagogy in the Training Experience of Italian Secondary School Teachers

from SSIS to FIT (and beyond?) (1998-2019)

Monica Ferrari

Training Secondary-School Teachers and the Position of the Minor.

Reflections from a Juridical-Constitutional Perspective

Giuditta Matucci 
The Role of Pedagogy in the Initial Training of Teachers of the Italian Secondary School Today Anna Bondioli, Maurizio Piseri und Donatella Savio

\section{Deutschland}

Seminare - eine hybride Ausbildungsform (18. bis 19. Jahrhundert)

Sabine Reh und Joachim Scholz

Gymnasiallehrer im Vormärz (1830-1848):

Zwischen Wissenschaft und Lehrberuf

Margret Kraul

Geisteswissenschaftliche Pädagogik und

höhere Lehrerausbildung (1915-1960)

Eva Matthes

Modernisierung durch Feminisierung? Zur Geschichte der

Lehrerinnenbildung in Deutschland (19. und frühes 20. Jahrhundert)

Elke Kleinau

Die Lehrerausbildung in der Bundesrepublik seit 1990: die Gründung

der Schools of Education

Charlotte Röhner

Die Entpädagogisierung der Lehrerbildung in der Bundesrepublik und die Entstehung der Bildungswissenschaft als Leitdisziplin in den 1990er Jahren

Rita Casale

Zur Heterogenität des Lehramtsstudiums in Deutschland:

Interaktionsanalysen universitärer Lehrkulturen (21. Jahrhundert)

Imke Kollmer, Hannes König, Thomas Wenzl und Andreas Wernet

Sexuelle Bildung: Geschichtliche und curriculare Perspektiven in der Lehrer/innenbildung

Julia Kerstin Maria Siemoneit und Jeannette Windheuser

Entwicklungen der Lehrerbildung in Deutschland

Jürgen Oelkers 


\title{
Geisteswissenschaftliche Pädagogik und höhere Lehrerausbildung (1915-1960)
}

\author{
Eva Matthes
}

\section{Einleitung}

Die geisteswissenschaftlichen Pädagogen Eduard Spranger, Herman Nohl, Theodor Litt und auch ihre bekanntesten Schüler Erich Weniger und Wilhelm Flitner ${ }^{1}$ haben sich seit 1915 bis in die 1960er Jahre in einer Reihe von Denkschriften und Publikationen zu Fragen der Lehrerbildung geäußert und auf die Entwicklung derselben massiv Einfluss zu nehmen gesucht. Im Blick auf die Volksschullehrerbildung ging es hierbei vor allem um die Frage ihrer Akademisierung und in diesem Kontext um den angemessenen Ausbildungsort sowie um den spezifischen Aufgabenzuschnitt des Volkslehrers als Beauftragten des Staates (nicht einer Religion/ Konfession oder einer Partei).

Der Fokus dieses Beitrags liegt allerdings auf den - selteneren - Stellungnahmen der geisteswissenschaftlichen Pädagogen zur ,höheren Lehrerbildung'. Sie verfolgten bei ihren Beiträgen - bei aller Unterschiedlichkeit im Detail, auf die später noch einzugehen sein wird - folgende gemeinsame Zielsetzungen:

Erstens strebten die geisteswissenschaftlichen Pädagogen die institutionelle Trennung der Ausbildung der Volksschullehrer und der höheren Lehrer an. Den Ort der Ausbildung der Volksschullehrer sahen sie an Pädagogischen Akademien, einer spezifischen Einrichtung im Preußen der 1920er Jahre, beziehungsweise an Pädagogischen Hochschulen, denjenigen der höheren Lehrkräfte an den Philosophischen Fakultäten der Universitäten. Pädagogische/ Erziehungswissenschaftliche Fakultäten an Universitäten lehnten sie entschieden ab.

Die pädagogische Wissenschaft sollte zweitens als eigenständige Disziplin in der Lehrerausbildung an den Universitäten verankert werden und Prüfungsstoff in der ersten Staatsprüfung sein, nicht erst nach Abschluss des Referendariats.

Drittens wurde angestrebt, die pädagogische Wissenschaft in der höheren Lehrerausbildung als Kulturpädagogik zu etablieren, die sich hermeneutisch und phänomenologisch mit der Rolle von Erziehung und Bildung im kulturellen Ganzen beschäftigt. Der geschichtlichen Perspektive wurde eine zentrale Rolle für das Verständnis und die normative Orientierung der Gegenwart zugeschrieben.

Die geisteswissenschaftlichen Pädagogen zielten viertens darauf, die philosophischen beziehungsweise geisteswissenschaftlichen Fakultäten der Universitäten von anwendungsorientierten, technizistischen und rezeptologischen Sichtweisen reinzuhalten, die pädagogische Wissenschaft etwa auf Handwerk oder Methode reduzierten. Vor diesem Hintergrund lehnten die geisteswissenschaftlichen Pädagogen auch - in klarer Abgrenzung zu den Herbartianern

$1 \mathrm{Zu}$ ihren Biografien und ihrer Verwurzelung im geisteswissenschaftlichen Denken im Anschluss an Dilthey s. Matthes 2009, S. 3ff. 
und in Frontstellung zu den experimentellen Pädagogen - an die Universitäten angeschlossene Übungsschulen für die höhere Lehrerbildung $a b$.

Fünftens sollte verhindert werden, die höhere Lehrerausbildung durch die Marginalisierung der Fachwissenschaften zu pädagogisieren. Alle geisteswissenschaftlichen Pädagogen unterstrichen die Bedeutsamkeit der Fachwissenschaften für eine inhaltlich niveauvolle, das erreichte kulturelle Niveau sichernde und dessen Weiterentwicklung ermöglichende Lehrertätigkeit.

Am ausführlichsten hat sich von den genannten Pädagogen Eduard Spranger mit Fragen der höheren Lehrerbildung beschäftigt und als Politikberater auf diese Einfluss zu nehmen gesucht. Deshalb wird er auch bei den folgenden Ausführungen im Zentrum stehen. Ergänzend werden Positionierungen Herman Nohls und des ebenfalls in Fragen der Lehrerbildung sehr aktiven Erich Wenigers vorgestellt.

\section{Eduard Sprangers Denkschrift aus dem Jahr 1915 - Hintergrund und zentrale Argumentationslinien}

Im Wintersemester 1910/11 übernahm Eduard Spranger die Professur für Philosophie und Pädagogik an der Universität Leipzig. Sein Vorgänger war der experimentelle Pädagoge Ernst Meumann, der nach nur zwei Semestern auf die Professur für Philosophie und Pädagogik am ,Allgemeinen Vorlesungswesen' in Hamburg gewechselt hatte. ${ }^{2}$ In seiner Antrittsvorlesung machte Spranger sein Verständnis von Pädagogik an der Universität deutlich, indem er sich von zwei Auffassungen abgrenzte: Zum einen davon, dass „die Pädagogik in der Philosophie aufgehe ", 3 zum anderen davon, dass die Pädagogik ihre Selbständigkeit aus ihrem Handlungsfeld ableite, da „die Pädagogik im Zusammenhang der philosophischen Problemstellung ihren ganz eignen Ursprung hat und daß sie nur dann ihre Selbständigkeit als Wissenschaft erringen kann, wenn sie sich ihres eigentümlichen methodischen Charakters und ihrer zunächst nur von der Philosophie aus bestimmbaren Aufgabe bewußt ist“. ${ }^{4}$

Diese Gedanken greift er wieder auf und führt sie noch genauer aus in einer Denkschrift für den preußischen Minister der geistlichen und Unterrichts-Angelegenheiten von Trott zu Solz im Juni 1915, die dieser von jenem erbeten hatte. Die Denkschrift ist ein Plädoyer für „die Pflege der wissenschaftlichen Pädagogik an den preußischen Universitäten "5 und enthält eine klare Ablehnung des von Trott zu Solz vorgelegten Entwurfs der Neuordnung der Prüfung für das höhere Lehramt. In dem war die Pädagogik in der wissenschaftlichen Prüfung am Ende des Universitätsstudiums - anders als in der bis dato gültigen Prüfungsordnung - nicht mehr vorgesehen. Pädagogische Inhalte sollten vielmehr am Ende der anschließenden zweijährigen praktischen Ausbildung abgeprüft werden. Spranger sieht darin eine klare Abwertung der Pädagogik und weist darauf hin, dass dadurch auch die pädagogischen Lehrveranstaltungen deutlich an Nachfrage verlören. ${ }^{6}$

Der Argumentationszusammenhang seiner Denkschrift ist folgender:

2 Vgl. hierzu Hopf 2004, S. $144 f f$.

3 Spranger 1910/1973, S. 222.

4 Ebd.

5 GstA PK, I. HA Rep. 76 Va Sekt. 1 Tit. VII Nr. 50 Bd. 1, Bl. 172r.

6 Vgl. Bl. 175 r. 
Er begründet zunächst die Notwendigkeit der pädagogischen Wissenschaft als Reflexionswissenschaft des „besonderen, sehr umfassenden Kulturgebiet(s)“ der „Erziehung und Menschenbildung“"? In diesem Zusammenhang wendet er sich gegen die - seit Friedrich August Wolf - überkommene Auffassung der Philologen, dass die pädagogische Wissenschaft nur „eine Sammlung von Rezepten“ sei; sie sei vielmehr „Kulturwissenschaft“ und habe als solche „eine enge Beziehung zu allen Wissenschaften, die von anderen Seiten des Kulturlebens - von Religion, Staat und Recht, Gesellschaft, Wirtschaft, Kunst und Sittlichkeit - handeln“. ${ }^{8}$ Einer so verstandenen pädagogischen Wissenschaft müsse nun im Studium der „höheren Lehrer" ein hohes Gewicht beigemessen werden, eine "theoretische Vorbereitung " ${ }^{\text {"9 }}$ af das praktische pädagogische Tun sei unverzichtbar. Die Philosophie, die nach wie vor als Prüfungsgegenstand in der wissenschaftlichen Prüfung vorgesehen war, sei hierfür nicht ausreichend. ${ }^{10}$ Es findet sich sogar die Formulierung, „daß die Pädagogik als Kulturwissenschaft, im wahrhaft akademischen Sinne betrieben, mindestens die gleiche allgemeinbildende und vertiefende Kraft besitzt wie die Philosophie“. ${ }^{11}$ Allerdings müsse sie hierfür eigenständig sein. Bisher seien die pädagogischen Lehrveranstaltungen unter ihrem Niveau geblieben, da die Pädagogik „meist ein Annex der Philosophie und für den Philosophen eine lästige Nebenaufgabe [sei], von der er sehr häufig selbst nichts versteht". ${ }^{12}$

Die Pädagogik an der Universität müsse eine theoretische, „über die allgemeinen Zusammenhänge und Faktoren der Erziehung überhaupt" reflektierende, keine „praktische Pädagogik“"13 sein, Übungsschulen (wie sie von den Herbartianern immer betrieben und auch von den experimentellen Pädagogen angestrebt wurden) seien deshalb abzulehnen. ${ }^{14}$ Spranger betont nachdrücklich, dass „die pädagogischen Vorlesungen an der Universität überhaupt nicht in die kleinste tägliche Praxis eingehen sollen und können. Ihr Wert liegt vielmehr darin, daß sie den Gesichtspunkt weiter nehmen als in der vereinzelten Schularbeit und ihren Anforderungen. Es ist geradezu ihr Verdienst, nicht zu praktisch zu sein, wie sonst die Anschauung erwächst, als ob Erziehung eine lehrbare und lernbare Technik wäre"15 - eine klare Spitze gegen die experimentelle Pädagogik und die mit ihr verbundenen Erwartungen.

Spranger schließt seine Denkschrift mit folgenden Forderungen:

„I. Beibehaltung der Pädagogik als Fach der Allgemeinen Prüfung neben Philosophie, und zwar im Sinne einer wissenschaftlichen Besinnung über den psychologischen, ethischen und kulturellen Geist der Erziehung (Pädagogik als Kulturwissenschaft, einschließlich der Kinderpsychologie) II. Erhebung der Pädagogik zum wählbaren ,Zusatzfach' der Prüfung [...], III. Initiative von seiten des Ministeriums ungeachtet des Widerstande der Fakultäten zur Förderung der Pädagogik an der Universität $[\ldots]^{“} \cdot{ }^{16}$

\footnotetext{
Bl. $172 \mathrm{v}$.

8 Bl. 172v: Herv. i. O.

9 Bl. 174r; Herv. i. O.

10 Bl. 174 r u. v.

11 Bl. $174 \mathrm{v}$.

12 Bl. $175 \mathrm{r}$ und $176 \mathrm{r}$.

13 Bl. 177 r.

14 Ebd.

15 Bl. 175v; Herv. i. O.

16 Bl. 181r.
} 
Seine Position zugunsten einer eigenständigen Kulturpädagogik an den Universitäten konnte Spranger erneut vertreten auf einer Konferenz des preußischen Ministeriums der geistlichen und Unterrichts-Angelegenheiten in Berlin im Mai 1917.

\section{Die Pädagogische Konferenz 1917}

Es ist hier nicht der Raum, ausführlich auf diese Konferenz einzugehen und die verschiedenen, teilweise auch widersprüchlichen Argumentationsmuster der Teilnehmer detailliert darzustellen. ${ }^{17}$ Die Gründe für die Konferenz waren vielschichtig; hier können nur die zentralen genannt werden: Zum Ersten waren von einer Reihe von Abgeordneten an den Kultusminister Bitten herangetragen worden, dass die Pädagogik an den Universitäten eine stärkere Vertretung erfahren solle, ${ }^{18}$ auch in den Tageszeitungen waren entsprechende Forderungen laut geworden. ${ }^{19}$ Zum Zweiten waren pädagogische Themenstellungen zentraler Bestandteil des öffentlichen Diskurses. Zum Dritten - und wohl sehr entscheidend - kannte der Minister nicht zuletzt durch die Denkschrift von Spranger den kulturpädagogischen Ansatz, der ihm für die notwendige nationalpädagogische Orientierung des deutschen Erziehungs- und Bildungswesens nach dem Krieg unverzichtbar erschien. Er kannte und teilte auch die Ängste gegenüber einer reformorientierten, sich mit sozialdemokratischen Kräften verbündenden Pädagogik, die sich nicht zuletzt auch für eine Akademisierung der Volksschullehrerbildung einsetzte, und sah eine Kulturpädagogik hierzu als Gegenmodell. ${ }^{20}$

Daran, dass die Pädagogik - in der richtigen, d.h. nationalpädagogischen Form (!) - Anspruch darauf hat, universitäres Fach zu sein, lässt der Kultusminister von Trott zu Solz keinen Zweifel, wenn er in seiner Einladung an die Professoren und Schulmänner schreibt: „Die Berechtigung der Pädagogik als Universitätsfach und die Reformbedürftigkeit der gegenwärtigen Übung werden dabei als anerkannt vorausgesetzt“ ${ }^{21}$ In seiner Begrüßung spricht er vom anerkannten „Heimatsrecht der Pädagogik an unsern Universitäten“"22 - eine Aussage, die durchaus einem Euphemismus gleichkommt.

Spranger bringt auf der Pädagogischen Konferenz seine kulturpädagogische Position erneut klar zum Ausdruck:

„Gegenstand der Pädagogik als Wissenschaft, die allein an die Universität gehört, ist der Bildungsvorgang als eine alle Gebiete der Kultur durchziehende Erscheinung. An diesem Bildungsvorgang sind als grundlegende Seiten zu unterscheiden: das Bildungsideal, die Bildsamkeit, der Bildner (Erzieher) und die Bildungsgemeinschaft (z.B. Schule)“.23

Auf der Basis dieses Viergespanns entfaltet Spranger das Curriculum einer wissenschaftlichen Pädagogik. ${ }^{24}$

17 Vgl. hierzu vor allem Schwenk 1977/78; Tenorth 2002; Matthes 2009.

18 Vgl. etwa GStA a. a. O. Bl. 183r.

19 Vgl. etwa Bl. 166r.

20 Vgl. hierzu Tenorth 2002, S. $196 f f$.

21 GstA Bl. 232r; Spranger 1960, S. 452.

22 Pädagogische Konferenz 1917, S. 8.

23 Schwenk 1977/78, S. 148.

24 Vgl. ebd. 
Noch die Handschrift des im Juli 1917 zurückgetretenen von Trott zu Solz trägt die am 31.07.1917 veröffentlichte Neufassung der Prüfungsordnung für das Lehramt an höheren Schulen Preußens, die am 1. April 1918 in Kraft trat, mit der die Prüfung der Pädagogik an das Ende der zweiten Phase verlegt wurde. ${ }^{25}$ Hier hatten auch die Denkschrift Sprangers von 1915, ein weiteres einschlägiges Gutachten Sprangers im Jahre 1916 und die kulturpädagogischen Argumentationen Sprangers auf der Pädagogischen Konferenz kein Umdenken bewirkt. Das einzige - strukturell marginale - Zugeständnis war, dass Pädagogik als „Zusatzfach“26 in der wissenschaftlichen Prüfung gewählt werden konnte. Die inhaltliche Füllung entsprach der kulturpädagogischen Position, ja, sie war wörtlich aus der Denkschrift Sprangers übernommen. Noch 1960 beklagt Spranger die Entscheidung der Verlegung der Pädagogikprüfung in die zweite Phase, die er auch in direkten Gesprächen mit dem zuständigen Beamten Reinhardt vergebens zu verhindern versucht habe. ${ }^{27}$

\section{Sprangers „Gedanken über Lehrerbildung“ (1920)}

Diese Schrift Sprangers hat ihre zentrale Zielsetzung in der Verhinderung einer universitären Volksschullehrerbildung. ${ }^{28}$ Deshalb entwickelt Spranger das Konzept einer „Bildnerhochschule“. ${ }^{29}$ Fragen der richtigen Gestaltung der Volksschullehrerbildung stehen also im Zentrum seiner Ausführungen. Er beschäftigt sich aber auch - quasi nebenbei - mit der höheren Lehrerausbildung an der Universität. Nachdrücklich wendet er sich gegen Tendenzen, aus der Philosophischen Fakultät „eine Lehrerfakultät“ zu machen. Seine Gegenargumente lauten: „Einmal muß es irgendwo im Staate eine Stelle geben, in der reine Wissenschaft, ohne Rücksicht auf nützliche Anwendungen, gesucht und gelehrt wird“. ${ }^{30}$ Mit nationalem Pathos fährt er fort:

„Diesen echt deutschen Gedanken, der sich seit dem 18. Jahrhundert durchgesetzt hat, dürfen wir uns nicht rauben lassen. Denn die geistige Größe des deutschen Volkes hat nicht zum geringsten Teil auf dieser Hingabe an zweckfreie Forschung beruht. Nur aus einem Überschuß an Wissen über die Bedürfnisse der direkten Verwertung hinaus folgen bisweilen ganz neue, ungeahnte Anwendungsmöglichkeiten oder kulturgestaltende Gedanken “. 31

Zum Zweiten müssten die angehenden höheren Lehrer mit den Früchten der wissenschaftlichen Erkenntnisse bekannt und vertraut werden, um diese in den höheren Schulen weitergeben zu können, deren Absolventen diese dann wieder in das Volk hinein vermittelten. ${ }^{32}$ Kritisch setzt er sich auch noch mit Behauptungen auseinander, dass

25 Vgl. Zentralblatt 1917, $\$ 1$.

26 Ebd., $\$ 25$.

27 Vgl. Spranger 1960, S. 453 f.

28 Vgl. Meyer-Willner 1986, S. 211-331.

29 Spranger 1920/1970, S. 53.

30 Ebd.

31 Ebd., S. 50f.

32 Vgl. ebd., S. $51 f$. 
„die Philosophische Fakultät sich künftig in zwei Stufen teilen werde: eine für die Berufsschulung von Lehrern und darüber eine Stätte der Forschung und rein wissenschaftlichen Weiterbildung. Ohnehin sei die Forschung in der Lehrerbildung an der Universität nur eine Fiktion“ “. ${ }^{33}$

Spranger stellt sich vehement gegen diese Auffassung: „Es ist aber gar nicht so, daß man Wissenschaft treiben könne nach Gefallen mit Forschung und ohne Forschung “. ${ }^{34}$ Vielmehr sei „jede echte Beschäftigung mit der Wissenschaft ein Erarbeiten“ und trage deshalb „den Charakter eines geistigen Produzierens [...] von den ersten Anfängen, die nur zur Erkenntnis des von anderen Erkannten führen, bis zu den Höhen selbständiger Neuentdeckungen “. ${ }^{35}$ Dies ist für Spranger auch das Kennzeichen einer wissenschaftlichen Lehrerbildung.

\section{Sprangers Denkschrift „Die Ausbildung der höheren Lehrer an der Universität" von 1925 (im Vergleich mit der Denkschrift von 1915)}

Spranger nimmt hier als Ausgangspunkt seiner Argumentation die „Ansicht“, „daß die Vorbildung der höheren Lehrer in den Philosophischen Fakultäten ihrer zukünftigen Berufsaufgabe nicht entspreche; ja, man hat gelegentlich behauptet, daß die Universität sie geradezu zu einer falschen Berufsauffassung erzöge" ${ }^{36}$ Spranger entgegnet nun, dass sich inzwischen die Mehrzahl der Universitätsprofessoren durchaus darüber im Klaren sei, dass sie ihre Lehre nicht allein auf die künftigen Forscher ausrichten dürfe, vielmehr die Bedürfnisse der künftigen höheren Lehrer berücksichtigen müsse. ${ }^{37}$

„Allerdings bedeutet diese Erkenntnis keinesfalls, daß die Philosophische Fakultät daraus für sich eine Umbildung folgern könnte, die als ihre ,Pädagogisierung' zu bezeichnen wäre. Sie kann weder formell ihren eigenen Lehrbetrieb ausschließlich an pädagogischen Grundsätzen orientieren, noch kann sie inhaltlich den pädagogischen Vorlesungen und Übungen so erheblichen Raum vergönnen, wie es heute vielfach gefordert wird“" ${ }^{38}$

Der Kern der Universität sei die „Wissenschaft als solche“. ${ }^{39}$ Darin sieht er kein Problem für die Lehrer der höheren Schule, denn auch an ihr stelle „das pädagogische Moment nur die Form“ dar, „in der sachliche Gehalte und objektive Bildungsgüter lebendig sein müssen, wenn die Erziehung nicht an geistiger Blutarmut zugrunde gehen soll“ ${ }^{40}$ Allerdings gebe es einige ernstzunehmende Probleme/Klagen: Dies sei zum einen die „Stoffdifferenz" ${ }^{\text {“4 }}$ zwischen Universität und höherer Schule. Hierbei müsse allerdings zunächst darauf hingewiesen werden, dass sich diese nicht grundsätzlich überwinden lasse; zwangsläufig müsse das „wissenschaftli-

33 Ebd., S. 71.

34 Ebd.

35 Ebd.

36 o.N. [Spranger] 1925. Spranger selbst hat in seinen „Gedanken über Lehrerbildung“ von den "teilweise recht anfechtbaren Leistungen der Universität“ (1920/1970, S. 69) auf dem Gebiet der höheren Lehrerausbildung gesprochen.

37 Vgl. o.N. [Spranger] 1925, S. 8.

38 Ebd.

39 Ebd.

40 Ebd., S. 9.

41 Ebd., S. 10. 
che Fundament “42 über das an der Schule benötigte Wissen hinausgehen. Allerdings dürfe zum einen nicht davon ausgegangen werden, dass alle Studierenden einen „Forscherdrang “43 aufwiesen; zum anderen zweiten müssten die Stoffgebiete, die im Schulunterricht eine Rolle spielen, im Universitätsstudium zumindest Berücksichtigung finden. Als Abhilfe schlägt Spranger deshalb zum Ersten eine „Stufengliederung “44 des Studiums und individuelle Studienberatung vor, ${ }^{45}$ zum Zweiten eine stärkere Berücksichtigung der schulischen Inhalte und Methoden bei den Naturwissenschaften, wo sich das Problem im besonderen Maße zeige. ${ }^{46}$ Eine weitere Klage sei die Ausbildung der künftigen höheren Lehrer zu „bloßen Spezialisten“. ${ }^{47}$ Spranger betont demgegenüber zunächst die Notwendigkeit der wissenschaftlichen Spezialforschungen. ${ }^{48}$ „Trotz dieses Zwanges zur Beschäftigung mit Einzelfragen wird die recht verstandene Wissenschaft nicht im bloßen Spezialismus versinken. Das Allgemeine der Wissenschaft liegt weniger im Resultat als in der Fragestellung“. ${ }^{49}$

„Für die Zwecke der Lehrerbildung wird sich daraus noch mehr als für den rein wissenschaftlichen Seminarbetrieb die Richtschnur ergeben, die Themata der Übungen so zu wählen, daß sie von einem einzelnen und begrenzten Ausgangspunkt weite Perspektiven eröffnen, und daß die Methode, die an diesem Objekt angewandt wird, typische und sinngemäß übertragbare Bedeutung für ein ganzes Gebiet, ja für die betreffende Wissenschaft überhaupt besitzt“ ${ }^{50}$

Als drittes stelle sich das Problem beziehungsweise die Herausforderung, dass der künftige höhere Lehrer „von früh an die Stelle kennen lerne, an der sich seine Wissenschaft der kulturellen Gesamtarbeit einordnet “. ${ }^{51}$ Dieser Einordnung müssten sich die einzelnen Wissenschaften stellen. ${ }^{52}$ Zusätzlich zur Schaffung eines kulturellen Bewusstseins müssten die Studien der Philosophie und der (Kultur-)Pädagogik beitragen. ${ }^{53}$ Wichtig in diesem Zusammenhang sei außerdem, die „Querverbindungen der Fächer" ${ }^{\text {54 }}$ zu beachten. Ein weiteres Augenmerk solle bei der höheren Lehrerausbildung an den Universitäten auf das notwendige „Können“ und die entsprechenden „Fertigkeiten" ${ }^{\text {" } 55}$ gelegt werden. So sei auf ein gutes Deutsch in Schriftsprache wie im Mündlichen, auf eine gute Vortragsweise, auf fremdsprachliche kommunikative Fertigkeiten sowie auf technische Fertigkeiten zu achten. ${ }^{56}$

Im Anschluss kommt Spranger noch auf eine besondere Differenz zwischen Universität und (höherer) Schule zu sprechen, die zwischen „reiner Wissenschaft und Bildung“. ${ }^{57}$ Diese Differenz sei nicht aufzuheben, allerdings könnten und sollten

42 Ebd.

43 Ebd., S. 11.

44 Ebd.

45 Vgl. ebd., S. 12.

46 Vgl. ebd., S. $13 f$.

47 Ebd., S. 14.

48 Vgl. ebd., S. 14 f.

49 Ebd., S. 16.

50 Ebd., S. 17.

51 Ebd., S. 18.

52 Vgl. ebd., S. $19 f$.

53 Vgl. ebd., S. $20 f$.

54 Ebd., S. 22.

55 Ebd., S. 23

56 Vgl. ebd., S. $23 f$.

57 Ebd., S. $24 f$. 
„die Universitätsdozenten auch die spezifisch bildenden Kräfte ihres Faches als solche fühlen und fühlen lassen; daß der besondere Bildungsgedanke, der ihrem Gebiete eigen ist, in ihnen lebe und daß sie gelegentlich diese Punkte berühren, an denen das strenge Eigengesetz der Wissenschaft mit dem organischen Wachstum und der zu formenden Totalität der Menschenseele zusammenhängt " ${ }^{58}$

Auch ein Plädoyer für Hochschuldidaktik beziehungsweise -pädagogik findet sich bei Spranger:

„Es wäre in jeder Beziehung sehr gut, wenn wir uns gewöhnten, gelegentlich beieinander zu hören und uns über unser Verfahren auszusprechen. Eine freie Gemeinschaft von Universitätslehrern, die für die [...] Mentalität der Studentengenerationen Interesse hätten und sich über hochschulpädagogische Fragen Gedanken machten, wäre auch für den besonderen Zweck der Lehrerbildung von großem Wert". 59

Mit Vorlesungen sollten Kolloquien verbunden werden. ${ }^{60}$

Das „Sondergebiet der pädagogischen Ausbildung "61 behandelt Spranger in seiner Denkschrift nur kurz. Er greift hierbei Gedanken auf, die er bereits in seiner Denkschrift 1915 geäußert hat und die er ebenfalls auf der ,Pädagogischen Konferenz' vom Mai 1917 zum Ausdruck brachte. Erneut setzt er sich für eine Verankerung einer Kulturpädagogik im höheren Lehramtsstudium ein. „Die Pädagogik [...] sollte in ihrem historischen Teil eine möglichst allseitige, philosophisch durchleuchtete Geschichte der Bildung und des Geistes, in ihrem systematischen Teil eine kulturphilosophisch begründete Theorie der Bildung sein“. ${ }^{62}$ Spranger betont 1915, wie bereits ausgeführt, dass die praktische Pädagogik an der Universität keinen Ort habe, 1925 etwas gemäßigter -, dass sie „nur unter zwei Gesichtspunkten zur Geltung kommen“ könne: „als notwendige Hinführung auf das künftige Berufsethos und als Gelegenheit, die persönliche Eignung oder Nichteignung für den Erzieherberuf rechtzeitig kennen zu lernen “. ${ }^{63}$ Auch seine in früheren Schriften geäußerte Ablehnung von Übungsschulen bleibt grundsätzlich bestehen:

„Von vornherein muß jede Diskussion über die Verbindung einer obligatorischen Übungsschule mit der Universität scharf abgelehnt werden [...] Gibt es an einer Universität einen Dozenten der Pädagogik, der der Praxis dauernd nahe genug steht, um sich und seinen geistesverwandten Mitarbeitern eine vorbildliche und anregende Wirksamkeit zuzutrauen, so mag er die Sache fakultativ ins Werk setzen". ${ }^{64}$

Schließlich stellt sich Spranger noch der Frage, „was die Universität zur Herausbildung des rechten Berufsethos im künftigen Lehrer tun könne " 65 und kommt zu folgendem Ergebnis: „Das Theoretische hat die pädagogische Vorlesung zu leisten; das tiefergreifende Praktische

58 Ebd., S. 25 f.

59 Ebd., S. 26.

60 Ebd.

61 Ebd.

62 o.N. [Spranger] 1925, S. 20f. Eine große Skepsis zeigt Spranger gegenüber fachdidaktischen Lehrveranstaltungen. Als Gründe hierfür führt er an: „der Student hat hierfür kaum Zeit, aber auch, weil ihm die praktische Berührung mit den Problemen fehlt, meist kein Verständnis“ (o. N. [Spranger] 1925, S. 28).

63 Ebd., S. 28f.

64 Ebd., S. 29.

65 Ebd., S. 30. 
das Vorbild des akademischen Lehrers, der von seiner Sache voll ist und sich für seine Schüler interessiert". ${ }^{66}$

Abschließend unterstreicht Spranger nochmals die Berechtigung der Ausbildung der höheren Lehrer an den Universitäten, auch mit ihren Mängeln: Es sei immer noch besser, „daß ein bloß gelehrter Kopf in die Schulen komme, als ein Kinderfreund, der ein absolut leerer Kopf ist und deshalb den Kindern nicht mehr geben kann, als schon jeder gleichaltrige Spielgenosse gibt “ ${ }^{\text {“67 }}$ - die Prioritätensetzung ist eindeutig.

\section{Positionierungen anderer geisteswissenschaftlicher Pädagogen (Nohl und Weniger)}

Herman Nohls Überlegungen zur höheren Lehrerbildung sind dem 1927 auf dem Göttinger Philologentag gehaltenen Vortrag ,Die Ausbildung der wissenschaftlichen Lehrer durch die Universität' zu entnehmen. In diesem Beitrag finden sich viele Parallelen zu den Gedanken Sprangers in der Denkschrift von 1925, aber doch einige explizite Unterschiede. Zunächst weist Nohl auf die alte Klage über die „unzureichende Ausbildung der höheren Lehrer auf der Universität" hin, die darauf zurückzuführen sei,

„daß der Student auf der Universität als Fachwissenschaftler zum Forscher ausgebildet wird - und zwar je besser seine Lehrer sind, um so strenger -, um dann bei Antritt seines Berufes zu merken, daß ihn da noch sehr andere Aufgaben erwarten als die Vermittlung des reinen Wahrheitssuchens". ${ }^{68}$

Dieser Widerspruch sei unaufhebbar und permanent virulent. Allerdings sei noch ein neues Problem hinzugekommen, dass nämlich die Inhalte, die von den Lehrern im Schulunterricht zu vermitteln seien, teilweise an der Universität nicht mehr gelehrt würden.

„Der Rückzug gegenüber der pädagogischen Forschung auf die Wissenschaftlichkeit des Lehrers kann jetzt nicht mehr helfen, weil diese Wissenschaftlichkeit selbst in ihrem Inhalt - nicht in ihrer formalen Haltung - augenscheinlich dem Leben gegenüber [...] nicht mehr ausreicht: man fühlt sich mit ihr nicht mehr sicher im Leben" ${ }^{69}$

Nohl führt das darauf zurück, dass die Wissenschaften zu lebensfern seien, ihren „Lebenssinn“70 nicht genügend entwickelten. Wie Spranger wendet er sich aber gegen eine Pädagogisierung der Wissenschaft, denn - eine Formulierung in Übereinstimmung mit Spranger - „die stärkste bildende Kraft der Wissenschaft liegt immer in der unbedingten sachlichen Energie, mit der sie ihrem eigenen Gesetz gehorcht " ${ }^{71}$ Dies müssten die wissenschaftlichen Lehrer stärker als es bisweilen der Fall sei zum Ausdruck bringen; damit würden sie auch die Lebensbedeutsamkeit ihrer Wissenschaft unterstreichen. ${ }^{72}$ Wie Spranger warnt Nohl davor, den Ausweg von der Enttäuschung durch die Fachwissenschaften in der Pädagogik zu suchen: Der Lehrer meine,

66 Ebd.

67 Ebd.

68 Nohl 1927/1929, S.183.

69 Ebd., S. 184.

70 Ebd.

71 Ebd.

72 Vgl. ebd. 
„aus dieser Glaubenslosigkeit an seinen ,Stoff in die Pädagogik flüchten zu können. Aber es ist klar, daß man endgültig nicht erziehen kann ohne den Glauben an den Gehalt der geistigen Welt, die man vermittelt. Die Pädagogik an sich bleibt im wesentlichen formal, die höhere Kraft des Lehrers stammt aus seiner geistigen Überzeugung “. 73

Wie Spranger schlägt Nohl eine organisatorische Veränderung des Studiums vor: die Unterscheidung - modern gesprochen - zwischen einem Grund- und einem Hauptstudium mit einer klaren Trennung von Einführungs- und Schlussvorlesungen. ${ }^{74}$ Außerdem plädiert er wie Spranger für eine „bessere Studienberatung “ 75

„Die Theorie von dem besten Schwimmenlernen, nämlich dadurch, daß man ins Wasser geworfen wird, die gerade die lebendigsten Universitätslehrer gern verwenden, mag einmal in einfacheren geistigen Verhältnissen richtig gewesen sein, angesichts der aufgewühlten See, die der Student heute durchqueren muß, reicht sie nicht mehr aus ${ }^{\text {“ }}{ }^{76}$

Im nächsten Schritt wendet sich Nohl der Pädagogik im höheren Lehrerstudium zu. Er tritt nachdrücklich für ein wissenschaftliches Studium der Pädagogik ein:

„Es ist ganz unmöglich, die pädagogische Einführung in das Ethos seines Berufes für den Lehrer erst von dem Referendarjahr zu erwarten und so die große Aufgabe der Universität, die Idee der Berufe zu vermitteln gerade an dieser Stelle aufzugeben und der Praxis zu überlassen. Der Sinn der pädagogischen Kollegs und Seminare wird darum auch nicht die Einführung in die pädagogische Technik sein, sondern zu allererst die Rechtfertigung der pädagogischen Arbeit vor sich selbst im Zusammenhang des geistigen Daseins, damit der junge Mensch weiß, welche Stelle er im Kreis des Ganzen einnimmt, und ihm das Eigenwesen der pädagogischen Grundhaltung gegenüber den fremden Anforderungen des Lebens gewiß wird“.77

Auf der Basis seines Theorie-Praxis-Verständnisses lehnt also auch Nohl jede pädagogische Rezeptologie entschieden ab, es geht ihm um das Studium einer wissenschaftlichen (Kultur-) Pädagogik. Inhaltlich fordert er - in Übereinstimmung mit Spranger - Veranstaltungen zur allgemeinen Pädagogik, zur Geschichte der Pädagogik, zur Pädagogik der Gegenwart und zur Kinderpsychologie und die Einrichtung von Stellen für das dafür notwendige Personal. ${ }^{78}$ Anders als Spranger - und in expliziter Abgrenzung zur Berliner Denkschrift - hält er eine „Didaktik der Fächer" ${ }^{\text {“79 }}$ für unverzichtbar. Es sei keine Frage, dass in der Fachdidaktik „die entscheidende Brücke zwischen Fachwissenschaft und Pädagogik geschlagen“ werde, „und daß erst solche Didaktik seines Faches die Ausbildung des künftigen Lehrers auf der Universität abschließt" ${ }^{80}$ Gemeint sei erneut keine Unterrichtstechnologie, sondern „eine Grundbesinnung über die pädagogische Formung der jeweiligen Wissenschaft, ihren Bildungssinn und die Grundhaltung, die sie gibt, Stoffauswahl und Aufbau des Lehrplans, Stellung des Lehrers in dem Bildungsprozeß zwischen Stoff und Jugend usw. “. 81

73 Ebd., S. $184 \mathrm{f}$.

74 Vgl. ebd., S. $185 f$.

75 Ebd., S. 186.

76 Ebd.

77 Ebd.; Herv. i. O.

78 Vgl. ebd., S. 186f.

79 Ebd., S. 187.

80 Ebd.

81 Vgl. ebd., S. 186f. 
Nachdrücklich weist Nohl die Idee zurück, die pädagogische Ausbildung der höheren Lehrer an den Pädagogischen Akademien vorzunehmen. ${ }^{82}$ Die Pädagogik als Wissenschaft müsse an der Universität angesiedelt sein und eine wichtige Stellung innerhalb des wissenschaftlichen Studiums der höheren Lehrer innehaben.

Wie Spranger lehnt Nohl Übungsschulen ab und spricht sich - wie jener - für „das mehrwöchige Hospitieren in den Ferien "83 aus. Ebenso wie jener tritt er auch für eine Abschlussprüfung der Pädagogik an der Universität ein und beklagt deren vorgenommene Abschaffung als Fehler. $^{84}$

Der Nohl-Schüler Erich Weniger äußert sich Ende der 1940er, Anfang der 1950er Jahre mehrfach in Gutachten und Denkschriften zu Fragen der höheren Lehrerausbildung und bezeichnet die pädagogische Ausbildung der höheren Lehrer als das „dornigste Problem“" ${ }^{\text {“5 }}$ der Lehrerausbildung. Festzustellen ist, dass er die universitäre Lehrerausbildung deutlich problematischer als Spranger und auch als Nohl sieht, aber doch für diesen Weg plädiert. Kritisch festzustellen sei allerdings, dass die meisten der höheren Lehrer „im Grunde Wissenschaftler sein “86 wollten. Sie hätten keine „Zuneigung "87 zu ihrem Beruf. Dies sei ein untragbarer Zustand. „Wir müssen unseren ganzen Besitz an theoretischen Einsichten einsetzen, um das praktische Ziel, nämlich die Menschen auf ihren Erzieherberuf vorzubereiten, zu erreichen “ ${ }^{88}$ Die Universität - an der die höhere Lehrerausbildung stattfindet - müsse sich der „doppelte[n] Aufgabe“ stellen, die Studierenden „innerlich für den erzieherischen Beruf zu gewinnen und zugleich mit wissenschaftlichen Mitteln auf die Wirklichkeiten, die sie erwarten, vorzubereiten “. ${ }^{89}$ Eine große Herausforderung und ein noch ungelöstes Problem stelle die „möglichst frühe Entscheidung über die erzieherische Eignung "90 der Studierenden dar. Die notwendige wissenschaftliche Vorbereitung auf den höheren Lehrerberuf verlange - neben dem fachwissenschaftlichen und parallel zu diesem ${ }^{91}$ - ein erziehungswissenschaftliches Studium. Hierbei gehe es darum, „das wissenschaftliche Verständnis der Erziehungswirklichkeit, des Bildungswesens, der Schulorganisation, der ihnen zu Grunde liegenden Ideen, Theorien, Zielsetzungen zu vermitteln“" ${ }^{92}$ Mit dieser Bestimmung der Erziehungswissenschaft steht Weniger durchaus in der Tradition von Spranger und Nohl. Er plädiert - neben der hermeneutischen - für eine phänomenologische Perspektive auf das Erziehungs- und Bildungssystem:

„Diese Wirklichkeit der Erziehung und des Schulwesens hat eine Struktur, die man kennen muß, wenn man verantwortlich aus eigener Entscheidung und nicht nur als Techniker in ihr arbeiten will [...]. Macht man sich diese Erkenntnismöglichkeiten nicht zu eigen, so bleibt man trotz aller fach-

82 Vgl. S. $186 f$.

83 Vgl. ebd., S. 188.

84 Vgl. ebd., S. $188 \mathrm{f}$.

85 Weniger 1953/1990, S. 107.

86 Ebd., S. $107 f$.

87 Ebd., S. 108.

88 Ebd.

89 Ebd.

90 Weniger 1950/1952, S. 476; vgl. auch 1953/1990, S. 109.

91 Weniger stellt sich in seiner Denkschrift von 1949 „Die pädagogische Ausbildung der wissenschaftlichen Lehrer durch die Universität" gegen Pläne, z.B. auch des Niedersächsischen Kultusministeriums, das erziehungswissenschaftliche Studium der höheren Lehrer von der Universität an Pädagogische Hochschulen auszulagern (vgl. Weniger 1949/1952, S. 460ff.).

92 Weniger 1950/1952, S. 479. 
wissenschaftlichen Eignung bestenfalls ein Handwerker seines Faches, schlimmstenfalls ein Dilettant und Improvisator". ${ }^{93}$

Sehr deutlich formuliert er immer wieder seine Kritik an der mangelnden erziehungswissenschaftlichen Ausbildung: „Daß die Ausbildung der wissenschaftlichen Lehrer gerade in einem für den späteren Beruf wesentlichen Bereich den wissenschaftlichen Charakter vermissen läßt, gereicht ihr gewiß nicht zum Vorteil““. ${ }^{44}$ Außerdem macht sich Weniger - wie Nohl - für die Integration der Allgemeinen Didaktik und der Fachdidaktiken in das Studium der höheren Lehrer stark: ${ }^{95}$

„Leider findet sich gerade unter den Philologen noch vielfach der grollige [sic!] Aberglaube, daß die Fachwissenschaft die Entscheidung über ihre Bildungsbedeutung immer schon in sich trage, oder daß der Unterricht nur angewandte und vereinfachte Wissenschaft darstelle, daß der Stoff schon an sich bildende Wirkung habe, daß die Frage der Unterrichtsmethode eine rein praktische sei und was dergleichen handgreifliche, aber weit verbreitete Irrtümer mehr sein mögen. In Wahrheit liegen hier dornige Probleme, die umfassender Besinnung bedürfen, und zwar innerhalb jedes Faches [...], im Verhältnis der Fächer untereinander [...], in der Beziehung der Schulfächer zu den Wissenschaften und in der Frage des Wissenschaftscharakters der Schulfächer überhaupt “. ${ }^{96}$

Als sehr wichtig sieht es Weniger an, dass bei den Studierenden des höheren Lehramts „das pädagogische Ethos“ entwickelt werde, das sie mit den anderen Lehramtsstudierenden bzw. Lehrern teilen müssten und das in ihnen „das Gefühl für die innere Einheit der Erziehungsaufgabe unseres Volkes“ wachrufen müsse - trotz getrennter Ausbildungsgänge sollte sich nach Weniger also eine „innere Einheit ${ }^{\text {“97 }}$ zwischen den verschiedenen Lehrergruppen einstellen.

\section{Schlussbemerkungen}

Wenngleich uns die bei allen geisteswissenschaftlichen Pädagogen vorherrschende, in unterschiedlichem Ausmaß mit elitärem Beigeschmack versehene Auffassung einer sinnvollen institutionell getrennten Ausbildung von Grund-/Haupt-/Mittelschullehrern einerseits und Gymnasiallehrern andererseits doch sehr fremd geworden ist - und somit die in Baden-Württemberg noch existierenden Pädagogischen Hochschulen in den anderen 15 Bundesländern als Fremdkörper betrachtet werden -, erscheinen andere Überlegungen doch hochaktuell.

Das gilt zum einen für den Dauerbrenner der akademischen Lehrerbildung, dem sinnvollen Verhältnis zwischen Fachwissenschaften, Fachdidaktiken und Erziehungswissenschaften oder - wie es heute häufig heißt - Bildungswissenschaften (wobei dann meist nur noch die pädagogische Psychologie gemeint ist) und ihren jeweiligen Anteilen sowie möglichen Kooperationsformen im Lehramtsstudium.

93 Ebd., S. 479f.

94 Weniger 1949/1952, S. 459.

95 Hier nimmt Weniger eine klare Gegenposition zu Spranger ein. Allerdings weiß auch letzterer, dass der Lehrer nicht glauben dürfe, „wenn er nur die Wissenschaft habe, werde ihm alles andere von selbst zufallen“ (Spranger 1918, S. 250).

96 Weniger 1950/1952, S. 480.

97 Weniger 1949/1952, S. 466. 
Das gilt zum anderen für die Frage nach der inhaltlichen Füllung dessen, was im Lehramtsstudium in der wissenschaftlichen Pädagogik gelehrt und gelernt werden soll.

Gegenwärtig scheint es - auf der Basis internationaler Entwicklungen - an deutschen Universitäten (wieder) eine starke Tendenz zu geben, Pädagogik auf handwerklich/technisches Wissen zu beschränken und allgemeinpädagogische einschließlich historischer Fragestellungen gegenüber Themen wie ,Classroom Management', ,Feedbackkultur' und ,Diagnosekompetenz zu marginalisieren oder gar ganz zu eliminieren. Es wäre sicher aufschlussreich, die aktuellen Projekte der ,Qualitätsoffensive Lehrerbildung' des Bundesministeriums für Bildung und Forschung einmal auf diese Thematik hin zu analysieren.

Die geisteswissenschaftlichen Pädagogen setzten sich für eine eigenständige pädagogische Wissenschaft mit eigener Fragestellung ein, die - in Verbund mit in forschender Perspektive gelehrten Fachwissenschaften (und - teilweise - Fachdidaktiken) - zu Reflexions- und Kritikfähigkeit der angehenden Lehrkräfte gegenüber ihrem praktischen Tun führen und damit professionelles Handeln in einem umfassenden Sinne grundlegen sollte.

Es kann m. E. wahrhaft nicht schaden, sich daran wieder zu erinnern und sich sozusagen in modernisierter Weise in diese Tradition einzustellen.

\section{Quellen und Literatur}

\section{Ungedruckte Quellen}

Geheimes Staatsarchiv Preußischer Kulturbesitz (GStA PK), I. HA Rep. 76 Va Sekt. 1 Tit. VII Nr. 50: Die Vorlesungen und die Errichtung von Lehrstühlen für Pädagogik bei den Universitäten, März 1890 bis Januar 1921.

\section{Gedruckte Quellen}

Litt, Theodor (1918/1965): Eine Neugestaltung der Pädagogik. In: Ders.: Pädagogik und Kultur. Kleine pädagogische Schriften 1918-1926. Hg. v. Nicolin, Friedhelm. Bad Heilbrunn, S. 7-11.

Meumann, Ernst (1914): Abriss der experimentellen Pädagogik. Leipzig, Berlin.

Nohl, Herman (1927/21929): Die Ausbildung der wissenschaftlichen Lehrer durch die Universität. In: Ders.: Pädagogische Aufsätze. Langensalza, Berlin, Leipzig, S. 183-189.

Pädagogische Konferenz im Ministerium der geistlichen und Unterrichts-Angelegenheiten am 24. und 25. Mai 1917. Thesen und Verhandlungsbericht. Berlin.

Spranger, Eduard (1910/1973): Antrittsvorlesung [Die philosophischen Grundlagen der Pädagogik]. In: Ders.: Gesammelte Schriften II. Hg. v. Bollnow, Otto Friedrich /Bräuer, Gottfried. Heidelberg, S. 222-231.

Spranger, Eduard (1920/1970): Gedanken über Lehrerbildung. In: Ders.: Gesammelte Schriften Bd. III: Schule und Lehrer. Hg. v. Englert, Ludwig. Heidelberg, S. 27-73.

o. N. [Spranger, E.]: (1925): Die Ausbildung der höheren Lehrer an der Universität. Denkschrift der Philosophischen Fakultät der Friedrich-Wilhelms-Universität Berlin. Leipzig.

Spranger, Eduard (1918): Über die künftige Pflege der Pädagogik an den deutschen Universitäten. Gutachtliche Äußerungen zu der Pädagogischen Konferenz im Preußischen Ministerium der geistlichen und UnterrichtsAngelegenheiten am 24. und 25. Mai 1917. In: Zeitschrift für Pädagogische Psychologie und Experimentelle Pädagogik 19, H. 7/8, S. 209-234.

Weniger, Erich (1949/1952): Die pädagogische Ausbildung der wissenschaftlichen Lehrer durch die Universität. In: Ders.: Die Eigenständigkeit der Erziehung in Theorie und Praxis. Weinheim, S. 458-474.

Weniger, Erich (1950/1952): Pädagogische Ausbildung der Philologen. In: Ders.: Die Eigenständigkeit der Erziehung in Theorie und Praxis. Weinheim, S. 475-481.

Weniger, Erich (1953/1990): Aufgaben und Gestaltung der Lehrerbildung. In: Ders.: Lehrerbildung, Sozialpädagogik, Militärpädagogik. Politik, Gesellschaft, Erziehung in der geisteswissenschaftlichen Pädagogik. Ausgewählt u. kommentiert v. Gaßen, Helmut. Weinheim, Basel, S. 91-111.

Zentralblatt für die gesamte Unterrichtsverwaltung in Preußen (1917), S. 612-661. 


\section{Literatur}

Drewek, Peter (1996): Die Herausbildung der „geisteswissenschaftlichen“ Pädagogik vor 1918 aus sozialgeschichtlicher Perspektive. Zum Strukturwandel der Philosophischen Fakultät und zur Lehrgestalt der Universitätspädagogik im späten Kaiserreich und während des Ersten Weltkriegs. In: Zeitschrift für Pädagogik, 34. Beiheft: Die Institutionalisierung von Lehren und Lernen. Beiträge zu einer Theorie der Schule. Weinheim, Basel, S. 299-316.

Hopf, Caroline (2004): Die experimentelle Pädagogik. Empirische Erziehungswissenschaft in Deutschland am Anfang des 20. Jahrhunderts. Bad Heilbrunn.

Horn, Klaus Peter (2003): Erziehungswissenschaft in Deutschland im 20. Jahrhundert. Zur Entwicklung der sozialen und fachlichen Struktur der Disziplin von der Erstinstitutionalisierung bis zur Expansion. Bad Heilbrunn.

Matthes, Eva (2000): Theologe - Philologe - Fachwissenschaftler - Pädagoge? In: Anregung. Zeitschrift für Gymnasialpädagogik 46, H. 5, S. 328-341.

Matthes, Eva (2009): Die Pädagogik konstituiert sich als universitäres Fach. In: Sandfuchs, Uwe/Link, Jörg-W./ Klinkhardt, Andreas (Hg.): Verlag Julius Klinkhardt 1834-2009. Verlegerisches Handeln zwischen Pädagogik, Politik und Ökonomie. Bad Heilbrunn, S. 81-94.

Matthes, Eva (2011). Geisteswissenschaftliche Pädagogik. Ein Lehrbuch. München.

Meyer-Willner, Gerhard (1986): Eduard Spranger und die Lehrerbildung. Die notwendige Revision eines Mythos. Bad Heilbrunn.

Schwenk, Bernhard (1977/1978): Pädagogik in den philosophischen Fakultäten - Zur Entstehungsgeschichte der „geisteswissenschaftlichen Pädagogik in Deutschland. In: Jahrbuch für Erziehungswissenschaft 2: Wissenschaft im Reformprozeß/Aufklärung oder Alibi? Stuttgart, S. 103-131.

Spranger, Eduard (1960): Ernst Troeltsch über Pädagogik als Universitätsfach. In: Derbolav, Josef/Nicolin, Friedhelm (Hg.): Erkenntnis und Verantwortung. Festschrift für Theodor Litt. [mit Auszügen aus der „Pädagogischen Konferenz"] Düsseldorf, S. 451-463.

Tenorth, Heinz-Elmar (1989): Deutsche Erziehungswissenschaft im 20. Jahrhundert. In: Zedler, Peter/König, Eckart (Hg.): Rekonstruktionen pädagogischer Wissenschaftsgeschichte. Fallstudien, Ansätze, Perspektiven. Weinheim, S. $117-140$

Tenorth, Heinz-Elmar (2002): Pädagogik für Krieg und Frieden. Eduard Spranger und die Erziehungswissenschaft an der Universität Berlin 1913-1933. In: Horn, Klaus Peter/Kemnitz, Heidemarie (Hg.): Pädagogik unter den Linden. Von der Gründung der Berliner Universität im Jahre 1810 bis zum Ende des 20. Jahrhunderts. Stuttgart, S. 191-226. 